\title{
Neural Network Classifiers Using a Hardware-Based Approximate Activation Function with a Hybrid Stochastic Multiplier
}

\author{
BINGZHE LI and YAOBIN QIN, University of Minnesota \\ BO YUAN, Rutgers University \\ DAVID J. LILJA, University of Minnesota
}

\begin{abstract}
Neural networks are becoming prevalent in many areas, such as pattern recognition and medical diagnosis. Stochastic computing is one potential solution for neural networks implemented in low-power back-end devices such as solar-powered devices and Internet of Things (IoT) devices. In this article, we investigate a new architecture of stochastic neural networks with a hardware-oriented approximate activation function. The newly proposed approximate activation function can be hidden in the proposed architecture and thus reduce the whole hardware cost. Additionally, to further reduce the hardware cost of the stochastic implementation, a new hybrid stochastic multiplier is proposed. It contains OR gates and a binary parallel counter, which aims to reduce the number of inputs of the binary parallel counter. The experimental results indicate the newly proposed approximate architecture without hybrid stochastic multipliers achieves more than $25 \%$, $60 \%$, and $3 \mathrm{x}$ reduction compared to previous stochastic neural networks, and more than $30 \mathrm{x}, 30 \mathrm{x}$, and $52 \%$ reduction compared to conventional binary neural networks, in terms of area, power, and energy, respectively, while maintaining the similar error rates compared to the conventional neural networks. Furthermore, the stochastic implementation with hybrid stochastic multipliers further reduces area about $18 \%$ to $80 \%$, power from $15 \%$ to $113.1 \%$, and energy about $15 \%$ to $131 \%$, respectively.
\end{abstract}

CCS Concepts: • Hardware $\rightarrow$ Arithmetic and datapath circuits; Application specific integrated circuits;

Additional Key Words and Phrases: Stochastic computing, approximate activation function, neural networks

ACM Reference format:

Bingzhe Li, Yaobin Qin, Bo Yuan, and David J. Lilja. 2019. Neural Network Classifiers Using a Hardware-Based Approximate Activation Function with a Hybrid Stochastic Multiplier. f. Emerg. Technol. Comput. Syst. 15, 1, Article 12 (January 2019), 21 pages.

https://doi.org/10.1145/3284933

\footnotetext{
This work was supported in part by National Science Foundation grant no. CCF-1408123. Any opinions, findings and conclusions or recommendations expressed in this material are those of the authors and do not necessarily reflect the views of the NSF. Portions of this work were presented in the 35th IEEE International Conference on Computer Design [18]. Authors' addresses: B. Li, Y. Qin, and D. J. Lilja, University of Minnesota, 200 Union Street SE, Minneapolis, MN 55455; emails: \{lixx1743, qinxx143, lilja\}@umn.edu; B. Yuan, Rutgers University, 96 Frelinghuysen Road, Piscataway, NJ 08854; email: bo.yuan@soe.rutgers.edu.

Permission to make digital or hard copies of all or part of this work for personal or classroom use is granted without fee provided that copies are not made or distributed for profit or commercial advantage and that copies bear this notice and the full citation on the first page. Copyrights for components of this work owned by others than ACM must be honored. Abstracting with credit is permitted. To copy otherwise, or republish, to post on servers or to redistribute to lists, requires prior specific permission and/or a fee. Request permissions from permissions@acm.org. (C) 2019 Association for Computing Machinery. 1550-4832/2019/01-ART12 \$15.00

https://doi.org/10.1145/3284933
} 


\section{INTRODUCTION}

Neural networks, which are designed as a computational model based on neurons, are becoming prevalent in many areas such as gaming [30] and cancer detection [9]. With growing interests in neural networks, people are beginning to focus on hardware implementations to achieve lowpower back-end applications rather than only being limited to software implementations. Previous works $[8,27]$ are implemented by Field Programmable Gate Arrays (FPGA). However, those implementations are restricted to mobile devices or the Internet of Things (IoT) due to their high power and large area. In those back-end implementations, power and area are the extremely important factors, for example, implementations that can take longer to compute a result but do not have much power available, or devices that harvest energy from the environment. However, with growth of the application complexity, the size of neural networks and their power consumption will increase tremendously. Therefore, the requirement of low power and small area will become more urgent in those implementations.

A new method called stochastic computing (SC) [5] was investigated by previous works [1, 2, $25,26]$, primarily targeting hardware systems with low power, small area, and high fault tolerance. First, in the stochastic computing domain, simple logic circuits can achieve many complex arithmetic operations. For example, an AND gate can achieve a multiplication operation; several finite state machines achieved by flip-flops can accomplish the tanh() function, exponential function, and other functions [21]. Secondly, stochastic computing using bit-streams to represent floating values increases fault tolerance abilities in hardware implementations. Therefore, according to previous works [1, 2, 22], stochastic computing is an opportune method to solve those area, fault tolerance, and power issues in the back-end neural network applications.

Prior work $[7,10,14,15,16,19,23,24]$ has explored stochastic neural networks using different designs. For example, Ji et al. [7] implemented a neural network with stochastic components for the activation function and multiplication only. The binary adders are inserted between two layers. Therefore, their work faced long latency issues due to the conversion between stochastic bit-streams and binary values. Li et al. $[15,16]$ proposed a stochastic restricted Boltzmann machine (RBM) implementation by using the simple OR gates to achieve the matrix multiplications. However, the OR adders face high error when there are a large number of inputs. Another work [10] derived an accurate tanh-based neuron and improved error rates of stochastic neural networks substantially by introducing the binary parallel counter. Two other types of works implemented deep stochastic convolutional neural networks (CNNs) with approximate parallel counters using bipolar [23] and unipolar [14, 24] encoding formats, respectively. Li et al. [19] investigated different activation functions by using stochastic computing such as rectified linear unit (ReLU), Sigmoid, and tanh functions. However, all of them use stochastic components to implement traditional activation functions, which limits further hardware optimization. Therefore, there are opportunities to design a hardware-oriented activation function to reduce neural network hardware cost further.

This article proposes a new stochastic neural network architecture with a hardware-oriented approximate activation function and a hybrid stochastic multiplier. The format of stochastic arithmetic components uses only unipolar encoding, which reduces hardware cost compared to previous bipolar format methods. In addition, the hardware-oriented approximate activation function can be totally hidden in hardware implementations and thus reduce the stochastic implementation complexity and hardware costs. Moreover, a newly proposed hybrid stochastic multiplier contains OR adders and a binary parallel counter, and aims to decrease the number of inputs of binary counters resulting in the reduction of hardware cost. In this article, three classical neural networks, multiple layer perceptron (MLP), RBM, and CNN, are implemented with the new stochastic structure. The results indicate that the newly proposed stochastic architecture with the approximate activation function reduces hardware cost significantly, while retaining similar recognition error 
rates compared to conventional deterministic neural network implementations. Moreover, the implementation with hybrid stochastic multipliers can further reduce the hardware cost. The main contributions of this article are summarized as follows:

- A new stochastic neuron with the a new hardware-oriented activation function is proposed. It not only obtains similar recognition error rates to the binary neuron with the traditional sigmoid activation function, but also further reduces hardware cost compared to previous stochastic neuron architectures.

- A hybrid stochastic multiplier is proposed to further decrease the hardware cost of a single neuron and thus reduce the total hardware cost of the whole neural network implementation.

- The stochastic MLP, RBM, and CNN with the newly proposed stochastic architecture achieve much less hardware cost than previous stochastic neural network implementations, and also have similar recognition error rates compared to deterministic neural networks.

This article is organized as follows: Section 2 introduces the background of stochastic computing. Section 3 provides the motivation of this work and demonstrates the neural networks stochastic implementation. The experimental results of neural network comparisons are discussed in Section 4. The conclusion is drawn in Section 5.

\section{STOCHASTIC COMPUTING BACKGROUND}

In this section, we introduce stochastic computing components that are used in the stochastic neural networks in Section 3.

\subsection{Stochastic Bit-Streams}

A stochastic bit steam is a way to express a floating-point value, which consists of a sequence of binary digits. The value expressed by a stochastic bit-stream is computed from the primary statistic of the bit-stream or the probability of any given bit in the stream being a logic " 1 ". Two types of encoding formats are used in stochastic bit-streams, unipolar and bipolar [2]. They can express a real number $x$ in $[0,1]$ or $[-1,1]$, respectively. For the unipolar coding format, the probability of having " 1 "s in a bit-stream $X$ is $\operatorname{Pr}(X)=x$. On the other hand, for the bipolar format, the probability of seeing " 1 "s in a bit-stream $X$ is $\operatorname{Pr}(X)=(x+1) / 2$ [20].

Furthermore, to express values which are larger than 1 or smaller than -1 , the scaled functions are shown in Equations (1) and (2) for the unipolar and bipolar representations, respectively.

$$
\begin{aligned}
& \operatorname{Pr}(X)=x / N \\
& \operatorname{Pr}(X)=\frac{x / N+1}{2},
\end{aligned}
$$

where $N$ is the scaling coefficient to make $x / N$ located in $[-1,1]$ or $[0,1]$.

\subsection{Addition}

Two types of stochastic additions for two encoding formats are introduced by Dickson et al. [4] and Qian et al. [28]. Dickson et al. used a simple OR gate to perform approximate addition for the unipolar format only.

$$
C=A o r B=A+B-A B
$$

A scaled addition using a simple standard Multiplexer (MUX) is proposed by Qian et al. [28]. This method is suitable for both unipolar and bipolar encoding formats. According to Equation (4), 


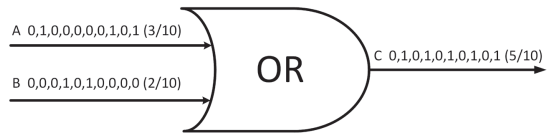

(a) OR gate for unipolar addition

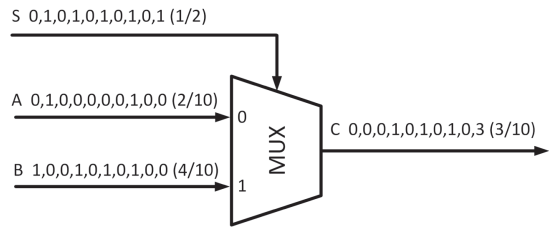

(b) MUX gate for scaled addition

Fig. 1. Stochastic addition with unipolar coding.

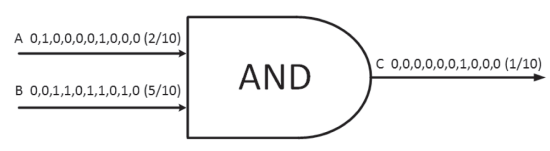

(a) Multiplication using AND for unipolar

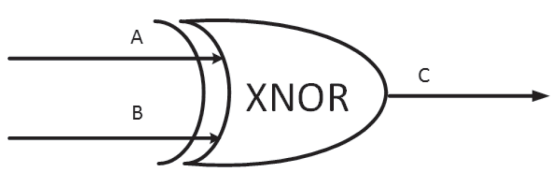

(b) Multiplication using XNOR for bipolar

Fig. 2. Stochastic multiplications for unipolar and bipolar.

when the select line of the MUX represents the value of 0.5 in the stochastic domain, the normal add operation is changed to $C=(A+B) / 2$, which scales the normal addition results down two times. Figure 1(b) shows an example of performing a scaled addition.

$$
C=A \cdot S+B \cdot(1-S)
$$

In order to generalize the simple two-input adder to a multi-input adder, Equations (3) and (4) are extended to Equations (5) and (6). In the ideal case for Equation (6), $N$ can be any positive integer. However, if we are restricted to only use 2-input MUXs in implemention of multi-input adder, $N$ should be a power of 2 .

$$
\begin{aligned}
& C=A_{1} \text { or } A_{2} \text { or } A_{3} \text { or ...or } A_{N} \\
& C=\frac{1}{N} \sum_{i=1}^{N} A_{i}
\end{aligned}
$$

\subsection{Multiplication}

Two types of multiplications are implemented by an AND gate for the unipolar (Figure 2(a)) and an Exclusive NOR (XNOR) gate for the bipolar representation of bit-streams (Figure 2(b)) [21].

As seen in Equations (7) and (8), suppose $A, B$, and $C$ are encoded as bipolar values of $a, b$, and $c$. Thus, $A=(1+a) / 2, B=(1+b) / 2$, and $C=(1+c) / 2$. Then, after passing an XNOR gate and simplifying Equation (8), we have $c=a b$.

$$
\begin{gathered}
C=A B+(1-A)(1-B) \\
\frac{c+1}{2}=\frac{a+1}{2} \frac{b+1}{2}+\left(1-\frac{a+1}{2}\right)\left(1-\frac{b+1}{2}\right)=\frac{a b+1}{2}
\end{gathered}
$$

\subsection{Sigmoid Function}

The sigmoid function is defined in Equation (9). Prior work has investigated stochastic sigmoid implementations. For example, Li et al. [15] proposed a stochastic sigmoid function from the stochastic $\tanh ()$ function using finite state machines as seen in Equation (10). 


$$
\begin{aligned}
S(x) & =\frac{1}{1+e^{-x}} \\
\frac{1}{1+e^{-x}} & =\frac{e^{x / 2}}{e^{x / 2}+e^{-x / 2}} \\
& =1 / 2\left(1+\frac{e^{x / 2}-e^{-x / 2}}{e^{x / 2}+e^{-x / 2}}\right) \\
& =\frac{1+\tanh (x / 2)}{2}
\end{aligned}
$$

Another work [19] used approximate parallel counters connecting finite-state machines to implement the sigmoid function. However, though both of their works can reasonably fit the curve of the sigmoid function, the performance of whole neural networks integrated with their work is inadequate. One reason for the bad recognition error rates is that the combination of matrix multiplication and sigmoid function does not perform well.

In this work, we proposed an approximate sigmoid function as seen in Equation (12). The newly proposed activation function is the bipolar format of $x / 2$. Therefore, we can ignore the activation function layer in our stochastic implementation while keeping the bit-stream format in the unipolar format. As the results show in Section 4, the deterministic neural networks keep almost the same recognition error rates when replacing the traditional sigmoid function by our approximate sigmoid function. The detailed introduction of the approximate activation function is provided in Section 3.2.

\section{STOCHASTIC NEURAL NETWORKS}

\subsection{Motivation}

Two major goals are pursued by stochastic neural networks, low hardware cost, and low recognition error rate.

Previous research $[10,14,15,17,19,23]$ focused on how to make stochastic implementations match original activation functions such as ReLU, tanh, and sigmoid in the stochastic domain. However, those hardware implementations limit further hardware optimization. Thus, reverse thinking: is there any activation function with an approximate format that can optimize stochastic hardware further?

Secondly, in stochastic computing, two encoding schemes are investigated for the ranges of $[0,1]$ and $[-1,1]$, respectively. Therefore, two formats are associated with different arithmetic computing components. For example, multiplication for unipolar format uses an AND gate and multiplication for bipolar uses an XNOR gate, which normally consists of three gates including AND and OR gates. As a result, AND gate multiplications achieve a smaller hardware cost than XNOR gate multiplications. Furthermore, in large neural networks, the difference of two types of multiplications will be amplified by an increasing number of neurons and has significant impacts on the total hardware cost of neural networks. Therefore, in this work, we only use unipolar format bit-streams in stochastic neural network implementations, which significantly reduces the hardware cost of neural network implementations compared to previous works.

Additionally, the three most popular activation functions in neural networks are ReLU, tanh, and sigmoid. Prior work $[10,14]$ has well investigated the error rates of stochastic neural networks with ReLU and tanh. Their implementations had similar recognition error rates to the deterministic neural networks. However, the sigmoid function has not been fully explored. Previous works $[16,19]$ implemented with the stochastic sigmoid function are still substantially worse than 
conventional implementations. Therefore, above discussions motivate us to design a new stochastic neuron architecture with lower hardware cost and lower recognition error rates compared to previous works.

\subsection{Approximate Sigmoid Function}

We propose a hardware-oriented approximate activation function, which can match well to stochastic neural networks. First, we extend the sigmoid function using Taylor series as seen in Equation (11). Since most of neural network coefficients are operated in the range of $[-1,1]$, the terms with high degrees will be around zero and can be ignored. Therefore, if we get rid of the terms with degrees higher than 2 , the first two terms are left and the equation becomes $\frac{1+x / 2}{2}$. Then, the proposed approximate sigmoid function is defined in Equation (12).

$$
\begin{aligned}
& \frac{1}{1+\exp (-x)} \approx 1 / 2+x / 4-x^{3} / 48+x^{5} / 480 \ldots \\
& y=\left\{\begin{array}{l}
1, \quad x>2 \\
1 / 4 * x+1 / 2, \quad-2 \leq x \leq 2 \\
0, \quad x<-2
\end{array}\right.
\end{aligned}
$$

By observation, $\frac{1+x / 2}{2}$ is the bipolar format of $x / 2$ in stochastic computing. Suppose a stochastic function can convert bit-streams from the unipolar format to bipolar format. The unipolar format of $x / 2$ after going through such a function becomes the bipolar format of $x / 2$, which is the unipolar format of the approximate activation function in Equation (12). Therefore, activation function layers can be totally ignored if a stochastic neuron has an output format like $\frac{1+x / 2}{2}$. In other words, with an output style of $\frac{1+x / 2}{2}$ in a neuron, we can totally ignore the activation function layers by keeping bit-stream formats as the unipolar format throughout the whole neural network. Such function is handled in our stochastic matrix multiplication without extra hardware implementation as discussed in Section 3.4. Thus, the architecture with omitted activation function layers reduces the hardware cost of stochastic neural networks. Moreover, for very deep neural networks, like the 19 layers in VGG Net [31] and 22 layers in GoogleNet [33], our implementation can obtain more benefits. Additionally, though the training issue is out of scope of this work, the newly proposed activation function provides an opportunity to solve the vanishing gradient issue of sigmoid function because its gradient has a constant value in the range of $[-2,2]$. As the results show in Section 4, the deterministic neural networks keep almost the same recognition error rates by replacing the conventional sigmoid function with the approximate activation function.

\subsection{Hybrid Stochastic Multiplier}

The matrix multiplier is the part that has the most hardware cost in the neural network implementations. In previous stochastic neural network implementations, there are two major implementations of matrix multiplications. One [16] is to use only AND gates and OR gates. The other one $[10,18,19]$ is to use AND gates to implement multiplication and then use parallel counters to sum up the products. However, though the first method achieves the extremely low hardware cost, its error rates are much higher than the other stochastic implementations. Compared to the first method, the second method obtains the much lower error rates but consumes higher hardware cost because of the binary parallel counters.

To reduce the hardware cost while keeping the low recognition error rates, we propose a hybrid structure for the stochastic matrix multiplication. As seen in Figure 3, before going into parallel counters, the products of bit-streams can be first partially summed up by OR gates, which decreases the number of inputs required for the binary parallel counters and thus reduces the total 


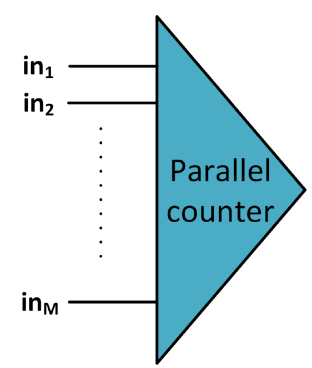

(a)

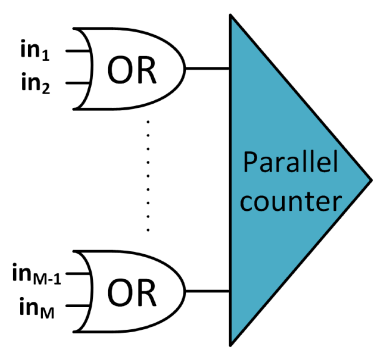

(b)

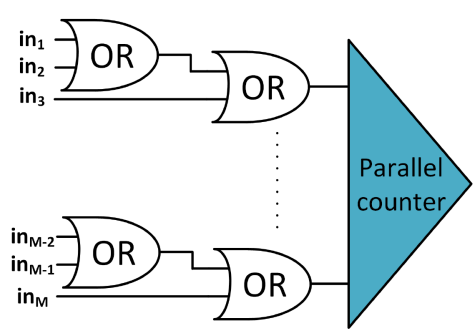

(c)

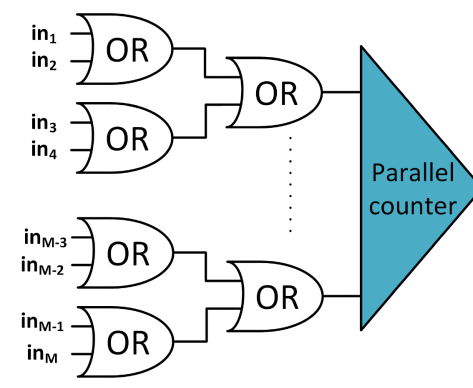

(d)

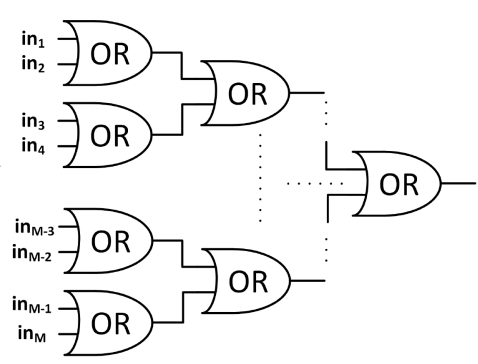

(e)

Fig. 3. Hybrid stochastic multiplier with different configurations of the OR adders. (a) Conventional parallel counter. (b) Hybrid structure with two-input OR adders $(\mathrm{N}=2)$. (c) Hybrid structure with three-input $\mathrm{OR}$ adders $(\mathrm{N}=3)$. (d) Hybrid structure with four-input OR adders $(\mathrm{N}=4)$. (e) Stochastic adder with only OR adders $(\mathrm{N}=\mathrm{M})[15]$.

hardware cost. Figure 3(b) to (d) indicates three OR-gate trees for partial addition. With increasing the number $(\mathrm{N})$ of inputs of the $\mathrm{OR}$ adders, the hybrid architecture will reduce the number of inputs of the binary parallel counter and thus reduce the hardware cost because OR gates are much cheaper than binary adders. To investigate the influence of the value of $\mathrm{N}$ on the error rates, we sum 128 non-zero values up with different stochastic hybrid adder structures in Figure 3 and compute their mean absolute errors (MAE). As shown in Figure 4, $\mathrm{N}=1$ corresponds to the results using the structure in Figure 3(a), N = 2 is for the structure in Figure 3(b), and $N=128$ is the results for the OR-tree structure [15] in Figure 3(e). Figure 4 indicates that the MAE is increased with increasing $\mathrm{N}$ and is also dependent on the bit-length. Moreover, for small values of $\mathrm{N}(\mathrm{N}<8)$, the hybrid structures obtain quite similar errors while reducing the hardware cost with increasing values of $\mathrm{N}$. When $\mathrm{N}$ reaches quite a large number like $\mathrm{N}=128$, the whole adder only uses OR gates in stochastic domain and the error is significantly increased. So, we obtain the same conclusion in Ref. [15].

In Section 4, we investigate the influence of the hybrid stochastic multiplier on the error rates of neural networks and hardware cost.

\subsection{Stochastic Neuron Implementation}

A single neuron is a basic unit in neural networks, which consists of a matrix multiplication and an activation function. In stochastic neural network implementations, matrix multiplication is a kernel component. On one hand, generally, it has a large number of additions that may cause large errors in the whole structure. On the other hand, it ensures correct transformations between two 


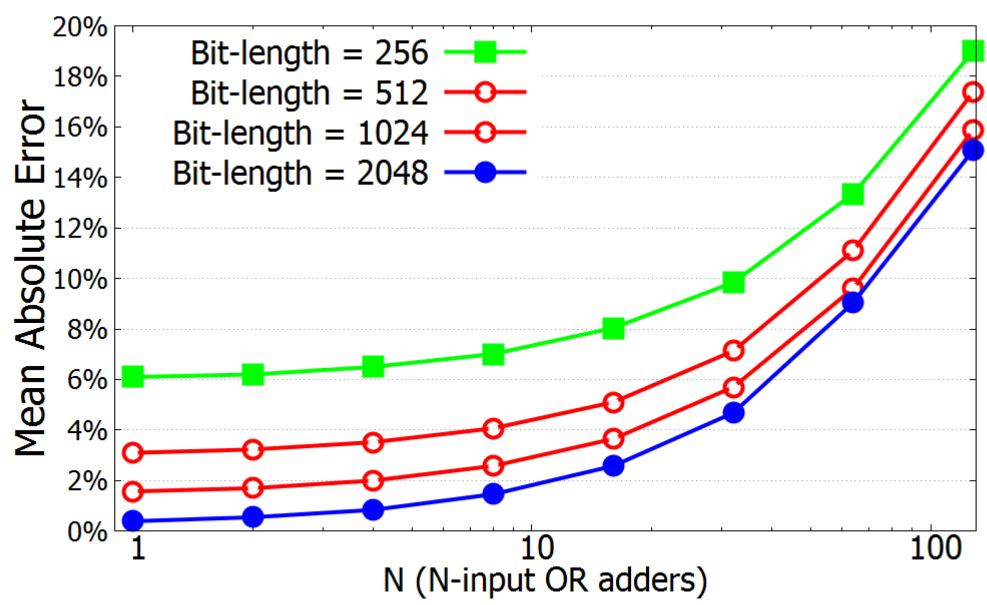

Fig. 4. Mean absolute errors with varying $\mathrm{N}$ from 1 to 128 ( $\mathrm{N}$ is the number of inputs of the OR adder) for bit-length from 256 to 2048.

layers. Thus, how to implement a stochastic neuron becomes significantly important for stochastic neural networks.

As discussed in Ref. [10], the multiplexer adder sacrifices precision because of scaling down. OR adders only can address input ranges located in [0,1]. Prior work [16] proposed a uni_pos_neg adder to implement such a large size of matrix multiplications based on OR adders. However, because OR adders are sensitive to correlation, implementations with OR gates have much worse recognition error rates than deterministic implementations. Other previous works $[10,23]$ implemented matrix multiplications by parallel counters with bipolar inputs and outputs. However, one weakness of their implementations is that the bipolar multiplication is implemented with more hardware cost than the unipolar multiplications as discussed in Section 3.1.

In this work, we propose a new stochastic neuron with matrix multiplications and an approximate activation function as seen in Figure 5. Generally, inputs of hybrid matrix multiplication in neural networks are from trained constant weights and image pixels (or activation function outputs). Since image pixel inputs and outputs of the sigmoid function are always in the range of $[0,1]$, the sign of each input bit-stream in Figure 5 only depends on signs of trained weights, which are constant values in neural network classifiers. Therefore, numbers of inputs at the negative part and at the positive part are clearly known as $N$ and $M$ in Figure 5 once neural networks have been trained. First, we scaled down constant weights four times before they passed through stochastic random bit-stream generators [11]. Then, according to the signs of constant weights, we separate products of inputs and weights into positive and negative parts. The positive and negative parts have extra constant inputs, $1 / 2+$ bias $^{+} / 4$ and bias $^{-} / 4$, respectively, where bias $^{+}$and bias $^{-}$ are obtained from the training process as constant values here. After that, hybrid parallel counters sum positive and negative output values together. Finally, the output becomes a bit-stream going through a comparator.

As seen in Equations (13)-(15), $P_{i s}$ and $Q_{s j}$ are inputs of matrix multiplication. The product of $P_{i s} \cdot Q_{s j}$ is implemented by an AND gate. According to the signs of the trained weights, products of bit-streams are assigned to the positive or negative part. $A_{i j}$ and $B_{i j}$ are the results of hybrid parallel counters from the positive part and the negative part, respectively. By subtracting $A_{i j}$ from $B_{i j}$, the result in Equation (15) equals to the unipolar format of our proposed approximate sigmoid 


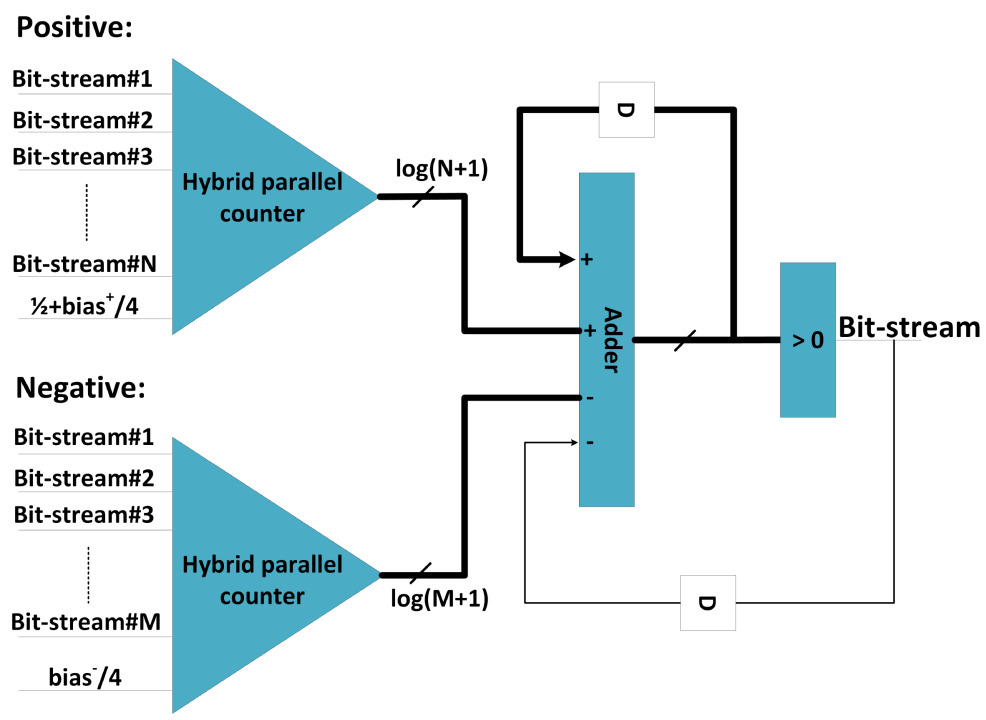

Fig. 5. The structure of a single neuron with matrix multiplication and approximate sigmoid function. Each bit-stream input is derived from an AND gate multiplication as seen in Figure 2(a). D is a delay element.

function in Equation (12).

$$
\begin{aligned}
A_{i j} & =1 / 4 * \sum_{\text {pos }} P_{i s} \cdot Q_{s j}+1 / 2+\text { bias }^{+} / 4 \\
B_{i j} & =1 / 4 * \sum_{n e g} P_{i s} \cdot Q_{s j}+\text { bias }^{-} / 4 \\
A_{i j}-B_{i j} & =\frac{1+1 / 2 *\left(\sum_{p o s}-\sum_{n e g}+\text { bias }^{+}-\text {bias }^{-}\right)}{2} \\
& =\frac{1+1 / 2 *\left(\sum P_{i s} \cdot Q_{s j}+\text { bias }\right)}{2}
\end{aligned}
$$

Therefore, only pre-operations are needed in this work like four times scaling down for trained weights and adding $1 / 2$ into bias $^{+}$. The newly proposed neuron does not have any specific hardware implementation for the activation function. Figure 5 indicates a single neuron structure with the newly proposed activation function. The format of inputs and outputs in this structure is the unipolar encoding.

\subsection{Multilayer Perceptron and Restricted Boltzmann Machine}

The multilayer perceptron [3] and restricted Boltzmann machine [6] are two types of neural networks. They share a common feature that nodes in one layer are fully connected to their next layers. In classifiers, the input layer corresponds to features of input images. Hidden layers correspond to dependencies of the image features. The output layer is a final decision function.

The stochastic implementations of the MLP and RBM are shown in Figures 6(b) and 7(b). According to stochastic arithmetic components, each stage of stochastic classifiers is well adaptable to bit-streams formats of its previous stage and its next stage. The nonlinear activation function is used with the approximate sigmoid function. Since overall formats of bit-streams use the unipolar 


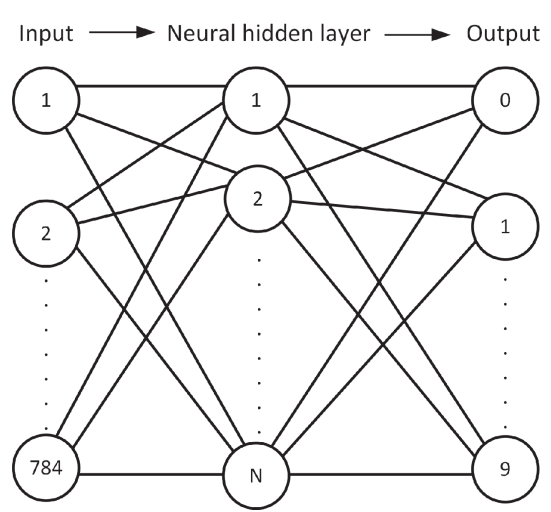

(a) Two layer MLP structure with $\mathrm{n}$ hidden neurons.

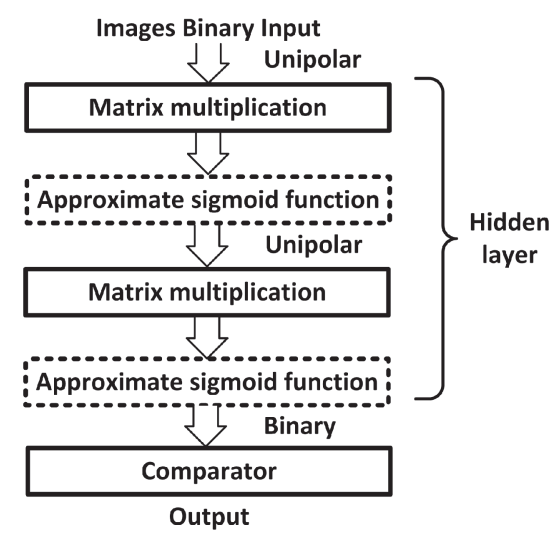

(b) The stochastic MLP classifier implementation data flow

Fig. 6. MLP structure and its stochastic dataflow.

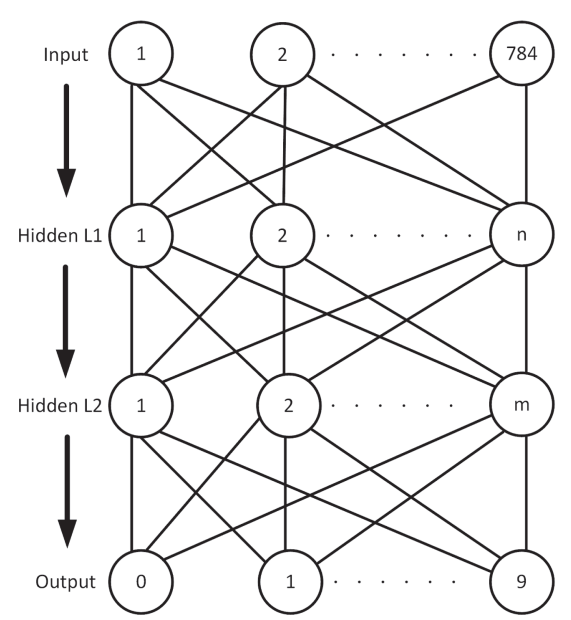

(a) Two layers restricted Boltzmann machine structure

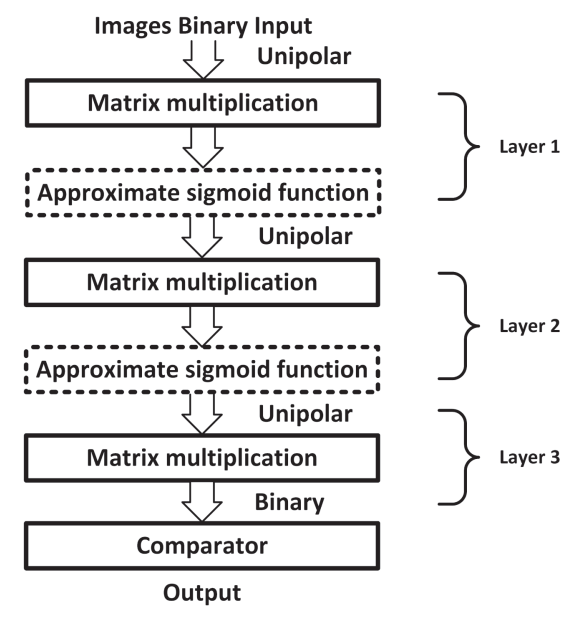

(b) The stochastic RBM classifier implementation data flow

Fig. 7. RBM structure and its stochastic dataflow.

format, layers of the activation function can be ignored as discussed in Section 3.2. Matrix multiplications are implemented by parallel counters with the unipolar format as seen in Figure 5.

\subsection{Convolutional Neural Network}

The CNN [12] normally contains three main types of layers, convolutional layer, pooling layer, and fully-connected layer. The pooling layer mainly focuses on down-sampling input data. A major operation in the pooling layer is to average neighbor data values or to obtain the maximum neighbor values. In this work, we use the average pooling to down-sample a $2 \times 2$ pixel region to one pixel. Therefore, we can use AND gates to multiply each input by $1 / 4$ and then sum them up to obtain their average value as seen in Figure 8. Matrix convolutional operation is quite similar 


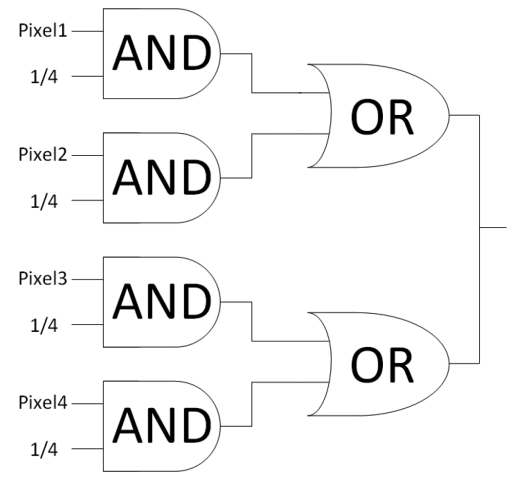

Fig. 8. Stochastic average pooling circuit implementation.

with matrix multiplication. The only difference is the orders of operated values. Therefore, we can use a similar circuit shown in Figure 5 to implement the matrix convolutional operation.

As seen in Figure 9(a), the CNN is built with a 784-1600-400-360-60-10 configuration. Figure 9(b) provides a dataflow of the CNN stochastic implementation. Output formats are perfectly matched to their next inputs. The activation function layers are hidden as discussed in Section 3.1.

\section{EXPERIMENTAL RESULTS OF NEURAL NETWORK COMPARISON}

In this section, all neural networks discussed in the previous sections are implemented as image classifiers. We use the MNIST handwritten digit image dataset [13], which consists of 60,000 training data and 10,000 testing data. All weights and coefficients of neural networks are trained and are regarded as constant values for the classifiers. Ten thousand testing data are used for testing stochastic and conventional neural networks. Recognition error rates and hardware costs of conventional and stochastic neural networks are discussed in the following sections.

\subsection{Recognition Error Rates}

To calculate recognition error rates, we ran our simulations for all stochastic neural networks with different bit-stream lengths to obtain a tradeoff between output error rates and required clock cycles.

4.1.1 MLP. We first investigated the MLP with different numbers of neurons with different bitlengths. Numbers of neurons $N$ shown in Figure 6(a) are varied from 32 to 512 . We compared error rates of stochastic and deterministic MLP implementations. In the deterministic implementation, MLPs with the sigmoid function and the approximate sigmoid function are provided. In the stochastic implementation, we compared our work with the MLP implementation in Ref. [15]. The bit-stream lengths are varied from 64 to 1,024 bits.

As seen in Table 1, two deterministic MLP implementations obtain similar error rates with different numbers of neurons. For the stochastic MLP, error rates are decreased with increasing bitstream lengths. Since computation time and accuracy of stochastic operations are directly proportional to the length of bit-streams [29], the longer bit-length means lower error rates, but longer computation time (clock cycles). Thus, the MLPs with the longest bit-length in Table 1 achieve the smallest error rates. Compared to previous stochastic implementations, our implementation derives much better error rates because the OR-tree MLP faces the higher issue for its OR adders than the binary parallel adders. The OR-tree based stochastic MLP loses recognition ability when bit-stream lengths are smaller than 512, while our implementation keeps less than $8 \%$ recognition 


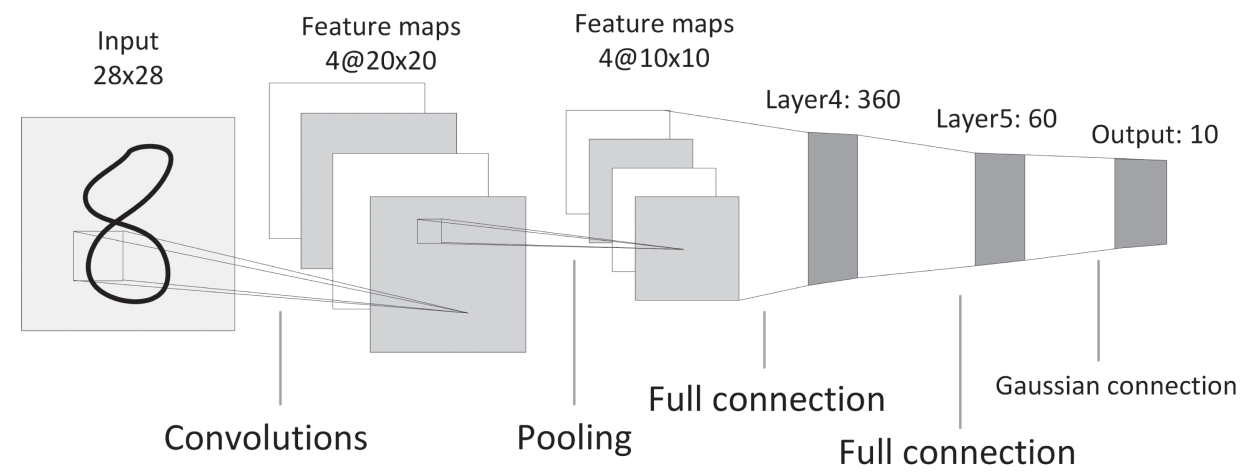

(a) A six layers CNN structure

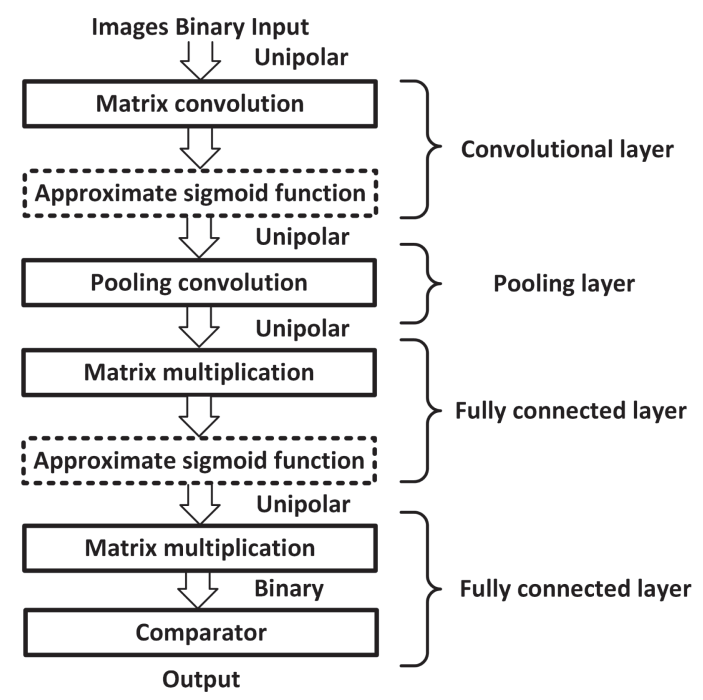

(b) The stochastic CNN classifier implementation data flow

Fig. 9. CNN structure and its stochastic dataflow.

error rates with the bit-stream length of 64 . When decreasing the bit-length to 32 , the implementation (binary parallel adder) loses the recognition ability. Therefore, as shown in Table 1, the stochastic MLPs are sensitive to the bit-length. With shorter bit-lengths, the recognition error rates increase significantly. Once the bit-length reaches to a specific level, the error rates will decrease slowly while increasing the bit-length until the error rates are saturated to the error rates of the binary deterministic MLPs. Therefore, the stochastic MLP implementations are quite sensitive to the bit-length when the bit-length is smaller than 512 and 64 for the OR-tree based implementation and our implementation, respectively. For the following neural network implementations including RBM and CNN, they obtain the similar conclusion that the stochastic neural networks are sensitive to the bit-length when it is quite small. After the bit-length is larger than a threshold value, the error rates of neural networks are becoming saturated. The threshold bit-length may be different for the neural networks based on the configurations and binary deterministic error rates 
Table 1. Recognition Error Rates Comparisons of MLP Classifiers with Different Bit-Lengths and Different Numbers of Neurons

\begin{tabular}{|c||c|cccccc|}
\hline \multirow{2}{*}{ \# of neurons } & $\begin{array}{c}\text { Deterministic with } \\
\text { sigmoid function }\end{array}$ & 32 & 64 & 128 & 256 & 512 & 1024 \\
\hline \hline 32 & $4.04 \%$ & N/A & N/A & $33.53 \%$ & $17.15 \%$ & $8.75 \%$ & $6.24 \%$ \\
64 & $3.07 \%$ & N/A & N/A & $34.37 \%$ & $19.00 \%$ & $8.64 \%$ & $5.60 \%$ \\
128 & $2.75 \%$ & N/A & N/A & $35.51 \%$ & $19.92 \%$ & $9.12 \%$ & $5.33 \%$ \\
256 & $2.43 \%$ & N/A & N/A & $32.84 \%$ & $10.56 \%$ & $8.41 \%$ & $5.35 \%$ \\
512 & $2.23 \%$ & N/A & N/A & $31.98 \%$ & $11.36 \%$ & $5.36 \%$ & $3.81 \%$ \\
\hline \hline \multirow{2}{*}{ \# of neurons } & Deterministic with the & \multicolumn{5}{|c|}{ This work (No OR adder) } \\
& approximate function & 32 & 64 & 128 & 256 & 512 & 1024 \\
\hline \hline 32 & $4.05 \%$ & $16.80 \%$ & $7.02 \%$ & $5.67 \%$ & $5.27 \%$ & $4.13 \%$ & $3.91 \%$ \\
64 & $3.07 \%$ & $14.27 \%$ & $4.11 \%$ & $3.41 \%$ & $3.23 \%$ & $3.06 \%$ & $3.06 \%$ \\
128 & $2.51 \%$ & $11.51 \%$ & $3.83 \%$ & $2.98 \%$ & $2.64 \%$ & $2.51 \%$ & $2.43 \%$ \\
256 & $2.46 \%$ & $23.06 \%$ & $5.30 \%$ & $2.93 \%$ & $2.63 \%$ & $2.54 \%$ & $2.54 \%$ \\
512 & $2.26 \%$ & $18.14 \%$ & $7.43 \%$ & $3.29 \%$ & $2.69 \%$ & $2.46 \%$ & $2.46 \%$ \\
\hline
\end{tabular}

Table 2. Recognition Error Rates Comparisons of MLP Classifiers with Hybrid Stochastic Multipliers

\begin{tabular}{|c|c|c|c|c|c|}
\hline \multirow[b]{2}{*}{ \# neurons } & \multirow[b]{2}{*}{$\mathrm{N}$-input $\mathrm{OR}$ adders } & \multirow[b]{2}{*}{ Deterministic } & \multicolumn{3}{|c|}{ Bit-length } \\
\hline & & & 64 & 128 & 256 \\
\hline \multirow{4}{*}{128} & None & \multirow{4}{*}{$2.51 \%$} & $3.83 \%$ & $2.98 \%$ & $2.63 \%$ \\
\hline & $\mathrm{N}=2$ & & $3.78 \%$ & $2.74 \%$ & $2.62 \%$ \\
\hline & $\mathrm{N}=3$ & & $3.89 \%$ & $2.77 \%$ & $2.67 \%$ \\
\hline & $\mathrm{N}=4$ & & $3.94 \%$ & $3.11 \%$ & $2.75 \%$ \\
\hline \multirow{4}{*}{256} & None & \multirow{4}{*}{$2.46 \%$} & $5.30 \%$ & $2.93 \%$ & $2.64 \%$ \\
\hline & $\mathrm{N}=2$ & & $5.22 \%$ & $2.89 \%$ & $2.71 \%$ \\
\hline & $\mathrm{N}=3$ & & $5.23 \%$ & $2.93 \%$ & $2.58 \%$ \\
\hline & $\mathrm{N}=4$ & & $4.97 \%$ & $3.08 \%$ & $2.61 \%$ \\
\hline
\end{tabular}

of the neural networks. Moreover, for the bit-length of 1024-bit, in our experiments, our method achieves about $2.46 \%-3.91 \%$ recognition error rates, which are quite similar to the deterministic implementations. However, the OR-tree based implementation only obtains $3.81 \%-6.24 \%$ error rates.

We additionally investigated the error rate by using hybrid matrix multiplications in Figure 3. As seen in Table 2, when increasing the number of inputs of the OR adders from $\mathrm{N}=2$ to 4, there is no influence on the recognition error rates. The reason is that OR gate performs the addition operation well with a small number of inputs. When increasing the number of adder inputs, the correlation between inputs becomes higher and causes the higher error rates. Li et al. [16] is the case that only uses OR gates without parallel counters (the number of inputs of the OR adders goes to a very large number) and obtains higher error rates than the parallel counter methods as seen in Table 1. Therefore, our proposed hybrid stochastic multipliers partially implement additions by OR gates which can reduce the hardware cost and keep the similar error rates with previous works. In summary, our stochastic MLP implementation achieves similar recognition error rates with the deterministic MLP implementation for all cases of when they have the same number of neurons. 
Table 3. Recognition Error Rates Comparisons of RBM Classifiers with Different Bit-Lengths

\begin{tabular}{|c||c|ccccc|}
\hline \multicolumn{1}{|c||}{ RBM structure } & Deterministic with & \multicolumn{5}{|c|}{ OR-tree RBM [16] } \\
& sigmoid function & 64 & 128 & 256 & 512 & 1024 \\
\hline \hline $784-100-200-10$ & $1.96 \%$ & N/A & N/A & $23.38 \%$ & $10.06 \%$ & $5.72 \%$ \\
$784-200-400-10$ & $1.35 \%$ & N/A & N/A & $18.83 \%$ & $7.93 \%$ & $4.24 \%$ \\
$784-300-600-10$ & $1.17 \%$ & N/A & N/A & $17.53 \%$ & $6.33 \%$ & $2.92 \%$ \\
$784-400-800-10$ & $1.10 \%$ & N/A & N/A & $16.69 \%$ & $5.17 \%$ & $2.37 \%$ \\
$784-500-1000-10$ & $0.98 \%$ & N/A & N/A & $16.53 \%$ & $5.10 \%$ & $2.32 \%$ \\
\hline \hline \multirow{2}{*}{ RBM structure } & Deterministic with the & \multicolumn{5}{c|}{ This work (No OR adder) } \\
& approximate function & 64 & 128 & 256 & 512 & 1024 \\
\hline \hline $784-100-200-10$ & $2.05 \%$ & $3.09 \%$ & $3.01 \%$ & $3.01 \%$ & $3.08 \%$ & $3.04 \%$ \\
$784-200-400-10$ & $1.34 \%$ & $1.88 \%$ & $1.90 \%$ & $2.07 \%$ & $2.04 \%$ & $1.88 \%$ \\
$784-300-600-10$ & $1.15 \%$ & $1.99 \%$ & $2.06 \%$ & $2.16 \%$ & $1.53 \%$ & $2.11 \%$ \\
$784-400-800-10$ & $1.07 \%$ & $1.59 \%$ & $1.58 \%$ & $1.69 \%$ & $1.73 \%$ & $1.88 \%$ \\
$784-500-1000-10$ & $1.01 \%$ & $1.49 \%$ & $1.53 \%$ & $1.53 \%$ & $1.45 \%$ & $1.46 \%$ \\
\hline
\end{tabular}

Table 4. Recognition Error Rates Comparisons of RBM Classifiers with Hybrid Stochastic Multipliers

\begin{tabular}{|c|c|c|c|c|c|c|}
\hline \multirow[b]{2}{*}{ \# neurons } & \multirow[b]{2}{*}{$\mathrm{N}$-input OR adders } & \multirow[b]{2}{*}{ Deterministic } & \multicolumn{4}{|c|}{ Bit-Length } \\
\hline & & & 32 & 64 & 128 & 256 \\
\hline \multirow{4}{*}{$784-500-1000-10$} & None & \multirow{4}{*}{$1.01 \%$} & $3.07 \%$ & $1.84 \%$ & $1.86 \%$ & $1.78 \%$ \\
\hline & $\mathrm{N}=2$ & & $2.73 \%$ & $1.55 \%$ & $1.52 \%$ & $1.47 \%$ \\
\hline & $\mathrm{N}=3$ & & $2.67 \%$ & $1.57 \%$ & $1.52 \%$ & $1.48 \%$ \\
\hline & $\mathrm{N}=4$ & & $2.59 \%$ & $1.51 \%$ & $1.46 \%$ & $1.43 \%$ \\
\hline
\end{tabular}

4.1.2 RBM. We investigated restricted Boltzmann machines with varying sizes from 784-100200-10 to 784-500-1000-10. We compared error rates of stochastic and deterministic RBM implementations. In the deterministic implementation, the RBM with the sigmoid function and the approximate sigmoid function are provided. In stochastic implementations, we compared our work with the RBM implementation in Ref. [15]. The bit-stream lengths are varied from 64 bits to 1,024 bits.

As seen in Table 3, the deterministic RBM implementation with the approximate sigmoid function has little difference of error rates compared to the RBM implementation with the conventional sigmoid function. However, in stochastic implementations, our proposed method improves error rates significantly compared to the OR-tree based stochastic RBM implementation. The OR-tree based stochastic RBM loses the recognition ability when the bit-stream lengths are smaller than 256, while our implementation remains less than $3.10 \%$ recognition error rates with bit-stream lengths varying from 64 to 1,024. In summary, for all sizes of RBM, our method derives about $1.45 \%$ to $3.09 \%$ recognition error rates, which are quite close to the deterministic implementations.

Moreover, to investigate the implementations with matrix multiplications, as seen in Table 4, with increasing the number of inputs of the OR adders from $\mathrm{N}=2$ to 4 , the implementation with hybrid stochastic multipliers achieves similar error rates as the implementation with the pure binary parallel counter. Since the neural networks have their special matrix patterns, it is possible that in some cases the structure with slightly larger $\mathrm{N}$ values $(\mathrm{N}<16)$ achieve a little lower error rates. Additionally, the trend will remain the same as shown in Figure 4 that the large $\mathrm{N}$ will cause 
Table 5. Recognition Error Rates Comparisons of CNN Classifiers with Different Bit-Lengths and Different Number of Inputs of OR Gates

\begin{tabular}{|c|c|c|c|c|c|}
\hline \multirow{2}{*}{\multicolumn{2}{|c|}{ CNN method }} & \multirow[b]{2}{*}{ Deterministic } & \multicolumn{3}{|c|}{ Bit-length } \\
\hline & & & 64 & 128 & 256 \\
\hline \multicolumn{2}{|c|}{ OR-based implementation [16] } & $1.39 \%$ & N/A & N/A & $19.35 \%$ \\
\hline \multicolumn{2}{|c|}{ Bipolar-based implementation [19] } & $2.99 \%$ & $6.06 \%$ & $5.58 \%$ & $4.34 \%$ \\
\hline \multirow{4}{*}{ our work } & None & \multirow{4}{*}{$1.41 \%$} & $2.12 \%$ & $1.57 \%$ & $1.49 \%$ \\
\hline & $\mathrm{N}=2$ & & $2.37 \%$ & $1.52 \%$ & $1.46 \%$ \\
\hline & $\mathrm{N}=3$ & & $2.13 \%$ & $1.60 \%$ & $1.44 \%$ \\
\hline & $\mathrm{N}=4$ & & $2.46 \%$ & $1.60 \%$ & $1.46 \%$ \\
\hline
\end{tabular}

$\mathrm{N}$ is the number of inputs of the OR adders.

larger error rates. The extreme case is that the OR-tree based RBM ( $\mathrm{N}$ is very large) in Table 3 obtains much larger error rates compared to our hybrid results $(\mathrm{N}=1$ to 4$)$ in Table 4.

4.1.3 CNN. We investigated three types of stochastic CNN implementations with sigmoid function. As seen in Table 5, our stochastic recognition error rates are quite similar error rates to the corresponding deterministic implementation. However, prior stochastic implementations obtain much worse error rates than their deterministic CNN implementations. Especially for the best case of our work, our stochastic recognition error rates are almost the same as the deterministic implementations. For instance, our 256-bit implementation obtains a $1.46 \%$ error rate compared to the $1.41 \%$ error rate of the deterministic implementation. To investigate the hybrid matrix multiplications, as seen in Table 5, the stochastic CNN implementation with hybrid stochastic multipliers obtains similar error rates as previous works.

\subsection{Hardware Cost}

We used the Synopsys Design Compiler to synthesize three typical neural networks with FreePDK $45 \mathrm{~nm}$ library [32] for the binary and stochastic implementations. The random number generators [11] are included in stochastic hardware synthesis results. The binary implementation uses 9-bit fixed binary.

4.2.1 Single Neuron Comparison. To investigate the hardware cost of different approaches in neural networks, we first explored the hardware cost of a single neuron because neurons are the basic units in neural networks. Therefore, the hardware cost of a single neuron can directly reflect the total hardware cost of neural networks. In stochastic technologies of neural networks, we can simply categorize the methods into three groups, OR-based neuron $[15,16]$, parallel counter bipolar (PC bipolar) based neuron [10,23] and parallel counter unipolar based neuron (this article). When considering the binary-based neuron, we compared four types of methods with different numbers of inputs in terms of area and power as seen in Figure 10(a) and (b). The OR-based neuron derives the least power consumption and area due to matrix multiplication only using OR adders. However, as shown in Section 4.1, the stochastic neural networks with OR-based neurons have much worse error rates compared to the neural networks with binary implementation and parallel counter implementation. Thus, we will not compare the OR-based neurons in the following hardware comparisons. Compared with the other two implementations, our work obtains about $47 \%$ area and $60 \%$ power reduction compared to the parallel counter bipolar-based neuron, and $43 \mathrm{x}$ area and 40x power reduction compared to the binary based neuron on average.

Moreover, we also investigate the hardware cost of a single neuron with hybrid matrix multiplications. As seen in Figure 11, we compare the different structures of hybrid matrix multiplications 


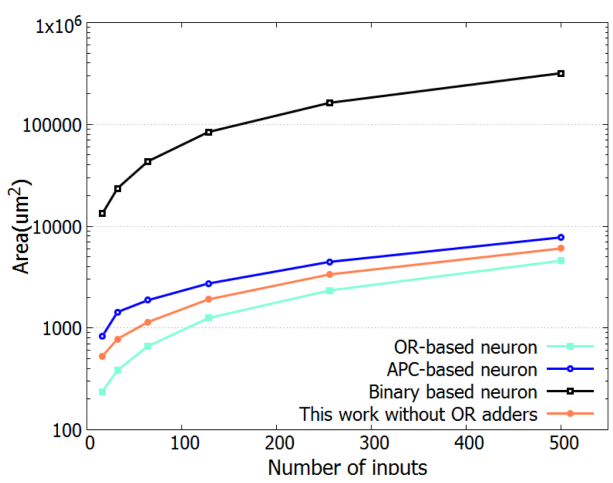

(a) Area

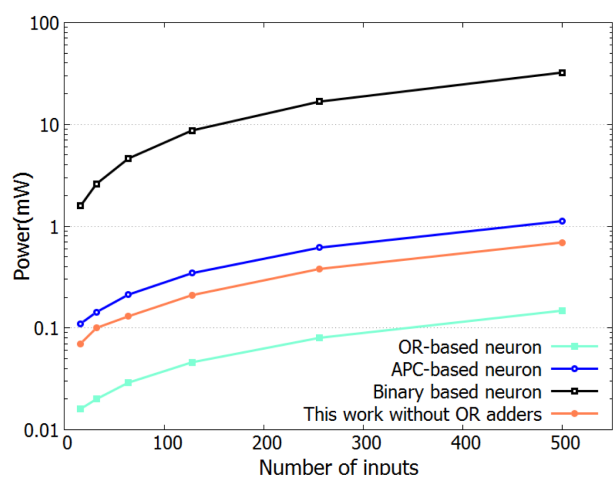

(b) Power

Fig. 10. Hardware cost comparison of a single neuron with a sigmoid function varying number of inputs.

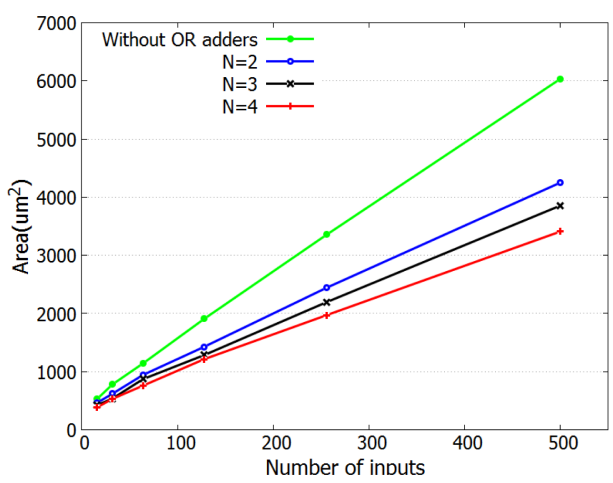

(a) Area

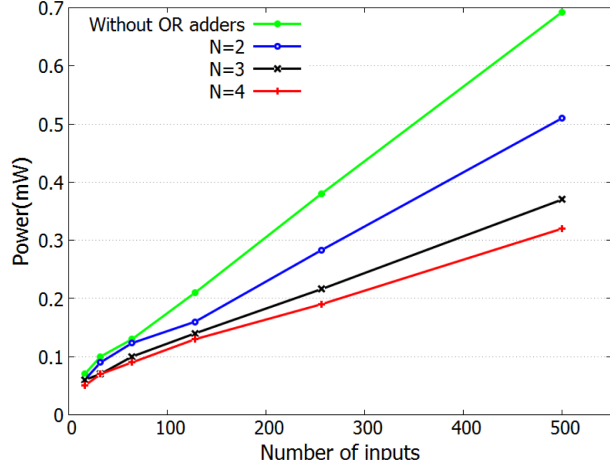

(b) Power

Fig. 11. Hardware cost comparison of a single neuron with different hybrid stochastic multipliers.

in Figure 3. When increasing the number of inputs of the OR adders in the hybrid matrix multiplications, the hardware cost of the neuron can be further reduced. As seen in Figure 11(a) and (b), compared to the pure parallel counter structure, varying the number of the input of the OR adders from 2 to 4 , the area and power cost can be further reduced by $13.8 \%-77 \%$ and $8.3 \%-118.4 \%$, respectively.

4.2.2 MLP. For hardware comparisons of MLPs, we vary the number of neurons from 32 to 512. Three methods are explored, binary, parallel counter bipolar, and parallel counter unipolar. As seen in Table 6, our work obtains about 50x area reduction and about 45x power consumption reduction compared to the binary implementation. Compared to the parallel counter bipolar implementation, we reduce about $25 \%$ area and about $60 \%$ power consumption. Additionally, with the bit-length of 64 , our work achieves about $60 \%$ and $3 x$ energy reduction compared to the binary implementation and previous stochastic neural network implementation, respectively. To investigate the hardware cost of the implementation with hybrid matrix multiplications as seen in Table 7, the implementation with hybrid matrix multiplications reduces the area about $44 \%-83 \%$, the power about $35.9 \%-131.2 \%$, and energy about $35.9 \%-131.2 \%$, respectively. Moreover, by increasing the number of inputs of the OR adder, the hardware cost can be reduced further. 
Table 6. MLP Hardware Comparison between Stochastic with 64 Bit-Length and Conventional MLP Implementation

\begin{tabular}{|c||c|c|c|c|}
\hline MLP & \# neurons & Area $\left(\mathrm{mm}^{2}\right)$ & Power $(m W)$ & Energy $(\mathrm{nJ})$ \\
\hline \hline & 32 & 16.07 & 1632.4 & 7.42 \\
Binary & 64 & 32.09 & 3258.9 & 14.83 \\
& 128 & 64.17 & 6512.7 & 29.63 \\
& 256 & 128.28 & 13,018 & 59.23 \\
& 512 & 256.55 & 26,033 & 118.45 \\
\hline & 32 & 0.40 & 59.04 & 13.99 \\
Stochastic & 64 & 0.78 & 115.17 & 27.29 \\
(PC bipolar) & 128 & 1.53 & 227.37 & 53.88 \\
& 256 & 3.03 & 451.79 & 107.07 \\
& 512 & 6.04 & 900.54 & 213.43 \\
\hline \multirow{5}{*}{ Stochastic } & 32 & 0.32 & 36.71 & 4.70 \\
(Our work) & 64 & 0.62 & 70.54 & 9.03 \\
& 128 & 1.20 & 138.41 & 17.72 \\
& 256 & 2.39 & 274.19 & 35.11 \\
& 512 & 4.76 & 545.67 & 69.86 \\
\hline
\end{tabular}

Table 7. MLP Hardware Comparison with Hybrid Stochastic Multipliers at Bit-Length $=64$

\begin{tabular}{|c|c|c|c|c|}
\hline \# neurons & N-input OR adder & Area $\left(\mathrm{mm}^{2}\right)$ & Power $(\mathrm{mW})$ & Energy $(\mathrm{nJ})$ \\
\hline \multirow{3}{*}{128} & None & 1.21 & 138.41 & 17.72 \\
\cline { 2 - 5 } & $\mathrm{N}=2$ & 0.84 & 101.81 & 13.03 \\
\cline { 2 - 5 } & $\mathrm{N}=3$ & 0.80 & 77.41 & 9.91 \\
\cline { 2 - 5 } & $\mathrm{N}=4$ & 0.66 & 60.79 & 7.78 \\
\hline \multirow{3}{*}{256} & $\mathrm{None}$ & 2.39 & 274.19 & 35.11 \\
\cline { 2 - 5 } & $\mathrm{N}=2$ & 1.65 & 200.97 & 25.73 \\
\cline { 2 - 5 } & $\mathrm{N}=3$ & 1.57 & 152.05 & 19.47 \\
\cline { 2 - 5 } & $\mathrm{N}=4$ & 1.31 & 118.61 & 15.19 \\
\hline
\end{tabular}

4.2.3 RBM. We explored different RBM sizes from 784-100-200-10 to 784-500-1000-10. In Table 8 , our proposed stochastic RBM obtains about 50x, 45x, and $52 \%$ reduction compared to the binary RBM implementation in terms of area, power, and energy. In addition, our work reduces about $30 \%$ area, saves power consumption about $63 \%$, and achieves about $2.5 \mathrm{x}$ energy reduction compared to the previous stochastic neural network implementation. Moreover, we investigate the hardware cost of implementation with different multipliers for the RBM with size of 784-500-100010 as seen in Table 9, the implementation with hybrid matrix multiplications reduces the area about $43 \%-79 \%$, the power about $35.5 \%-123.8 \%$, and energy about $35.5 \%-123.8 \%$, respectively. Moreover, by increasing the number of inputs of the OR adders, the hardware cost can be reduced further.

4.2.4 CNN. For the CNN hardware comparison, we compared with different implementations. As seen in Table 10, our implementation obtains $31 \mathrm{x}, 30 \mathrm{x}$, and $82 \%$ reduction compared to the binary CNN implementation and saves about $53.5 \%, 58.8 \%$, and $3.4 \mathrm{x}$ compared to the prior stochastic $\mathrm{CNN}$ with sigmoid function in terms of area, power, and energy, respectively. By applying the hybrid stochastic multiplier into the implementation, the new implementation achieves about $15.8 \%$, $12.8 \%$, and $36.5 \%$ reduction in terms of area, power, and energy, respectively. 
Table 8. Hardware Comparison between Stochastic RBM with 64 Bit-Length and Conventional RBM Implementation

\begin{tabular}{|c||c|c|c|}
\hline Neural networks & Area $\left(\mathrm{mm}^{2}\right)$ & Power $(\mathrm{mW})$ & Energy $(\mathrm{nJ})$ \\
\hline \hline RBM Binary 100-200 & 63.96 & $6,530.11$ & 29.71 \\
RBM Binary 200-400 & 152.40 & $15,569.3$ & 72.86 \\
RBM Binary 300-600 & 267.69 & $2,723.6$ & 122.96 \\
RBM Binary 400-800 & 406.65 & $41,365.7$ & 191.55 \\
RBM Binary 500-1000 & 570.54 & $58,022.7$ & 274.76 \\
\hline
\end{tabular}

Parallel counter bipolar implementation

\begin{tabular}{|c||c|c|c|}
\hline SC_RBM 100-200 & 1.67 & 234.15 & 55.49 \\
SC_RBM 200-400 & 3.87 & 550.09 & 127.84 \\
SC_RBM 300-600 & 6.59 & 949.87 & 204.58 \\
SC_RBM 400-800 & 9.86 & $1,436.77$ & 332.24 \\
SC_RBM 500-1000 & 13.74 & $2,015.55$ & 451.07 \\
\hline
\end{tabular}

Parallel counter unipolar implementation (our work)

\begin{tabular}{|c||c|c|c|}
\hline SC_RBM 100-200 & 1.27 & 143.46 & 18.41 \\
SC_RBM 200-400 & 2.91 & 329.92 & 37.69 \\
SC_RBM 300-600 & 5.01 & 571.25 & 62.20 \\
SC_RBM 400-800 & 7.60 & 867.09 & 111.02 \\
SC_RBM 500-1000 & 10.76 & $1,234.7$ & 180.26 \\
\hline
\end{tabular}

Table 9. RBM Hardware Comparison with Hybrid Stochastic Multipliers at Bit-Length $=64$

\begin{tabular}{|c|c|c|c|c|}
\hline RBM size & N-input OR adder & Area $\left(\mathrm{mm}^{2}\right)$ & Power $(\mathrm{mW})$ & Energy $(\mathrm{nJ})$ \\
\hline \multirow{3}{*}{$784-500-1000-10$} & None & 10.76 & $1,234.68$ & 180.26 \\
\cline { 2 - 5 } & $\mathrm{N}=2$ & 7.51 & 911.40 & 133.06 \\
\cline { 2 - 5 } & $\mathrm{N}=3$ & 6.96 & 673.63 & 98.35 \\
\cline { 2 - 5 } & $\mathrm{N}=4$ & 5.99 & 551.62 & 80.53 \\
\hline
\end{tabular}

Table 10. Hardware Comparison between Stochastic CNNs with 64 Bit-Length and Conventional CNN Implementation

\begin{tabular}{|c||c|c|c|}
\hline Neural networks & Area $\left(\mathrm{mm}^{2}\right)$ & Power $(\mathrm{mW})$ & Energy $(\mathrm{nJ})$ \\
\hline \hline Binary CNN & 122.25 & 13479 & 91.59 \\
Bipolar-based CNN & 5.91 & 740.94 & 172.20 \\
Our work & 3.85 & 439.53 & 50.21 \\
\hline
\end{tabular}

In summary, for stochastic implementations, there is a tradeoff between energy and recognition error rates. Generally, higher recognition error rates need longer bit-length and then cause larger energy consumption. Thus, by increasing bit-lengths, our implementation loses energy advantages compared to conventional binary implementations. When the bit-length is increased to 128-bit, our approach without hybrid stochastic multiplier achieves a little bit worse energy than the conventional binary implementations. However, our work will keep the same energy reduction compared to previous stochastic neural networks if stochastic neural networks use the same 
Table 11. CNN Hardware Comparison with Hybrid Stochastic Multipliers at Bit-Length $=64$

\begin{tabular}{|c|c|c|c|}
\hline N-input OR adders & Area $\left(\mathrm{mm}^{2}\right)$ & Power $(\mathrm{mW})$ & Energy $(\mathrm{nJ})$ \\
\hline None & 3.85 & 439.53 & 50.21 \\
\hline $\mathrm{N}=2$ & 3.24 & 379.63 & 43.37 \\
\hline $\mathrm{N}=3$ & 3.20 & 342.32 & 39.11 \\
\hline $\mathrm{N}=4$ & 3.03 & 321.89 & 36.77 \\
\hline
\end{tabular}

bit-lengths. Moreover, the tradeoff also happens between the hybrid stochastic multipliers. When selecting $\mathrm{N}$ with a large value as seen in Table 11, the implementation may reduce the recognition error rates slightly while reducing the hardware cost. Therefore, people can select the different $\mathrm{N}$ values depending on their requirements.

\section{CONCLUSION}

In this article, we explored three classical neural networks with newly proposed approximate activation function and hybrid stochastic multipliers. First, the approximate sigmoid function can make neural networks fit well to their stochastic implementation. Secondly, a newly proposed neuron with the hidden approximate activation function improves recognition error rates of stochastic neural networks further. As a result, the omitted activation function and unipolar-based architecture significantly reduce the hardware cost of neural networks. Thirdly, the newly proposed hybrid stochastic multiplier involves the OR adder and reduces the number of inputs needed for the binary parallel counter. Thus, the implementation with hybrid stochastic multiplier can further reduce the hardware cost of the whole implementation. According to our experimental results, our stochastic neural networks obtain similar recognition error rates with their binary implementation and better error rates than previous stochastic neural networks with the sigmoid activation function. Moreover, our stochastic implementation achieves more than $25 \%$ area reduction, $60 \%$ power saving, and 3x energy reduction than previous stochastic neural networks, and more than $30 \mathrm{x}, 30 \mathrm{x}$, and $52 \%$ reduction than the conventional binary neural networks in terms of area, power, and energy, respectively. Furthermore, the stochastic implementation with hybrid multiplier can reduce area from $18 \%$ to $80 \%$ and power from $15 \%$ to $113.1 \%$, respectively.

\section{REFERENCES}

[1] Armin Alaghi and John P. Hayes. 2013. Survey of stochastic computing. ACM Transactions on Embedded Computing Systems (TECS) 12, 2s (2013), 92.

[2] Bradley D. Brown and Howard C. Card. 2001. Stochastic neural computation. I. Computational elements. IEEE Transactions on Computers 50, 9 (2001), 891-905.

[3] Ronan Collobert and Samy Bengio. 2004. Links between perceptrons, MLPs and SVMs. In Proceedings of the 21st International Conference on Machine Learning. ACM, 23.

[4] Jeffery A. Dickson, Robert D. McLeod, and H. C. Card. 1993. Stochastic arithmetic implementations of neural networks with in situ learning. In Proceedings of the 1993 IEEE International Conference on Neural Networks. IEEE, 711-716.

[5] Brian R. Gaines et al. 1969. Stochastic computing systems. Advances in Information Systems Science 2, 2 (1969), 37-172.

[6] Geoffrey E. Hinton and Ruslan R. Salakhutdinov. 2006. Reducing the dimensionality of data with neural networks. Science 313, 5786 (2006), 504-507.

[7] Yuan Ji, Feng Ran, Cong Ma, and David J. Lilja. 2015. A hardware implementation of a radial basis function neural network using stochastic logic. In Proceedings of the 2015 Design, Automation \& Test in Europe Conference \& Exhibition. EDA Consortium, 880-883.

[8] Seul Jung and Sung su Kim. 2007. Hardware implementation of a real-time neural network controller with a DSP and an FPGA for nonlinear systems. IEEE Transactions on Industrial Electronics 54, 1 (2007), 265-271. 
[9] Murat Karabatak and M. Cevdet Ince. 2009. An expert system for detection of breast cancer based on association rules and neural network. Expert Systems with Applications 36, 2 (2009), 3465-3469.

[10] Kyounghoon Kim, Jungki Kim, Joonsang Yu, Jungwoo Seo, Jongeun Lee, and Kiyoung Choi. 2016. Dynamic energyaccuracy trade-off using stochastic computing in deep neural networks. In Proceedings of the 53rd Annual Design Automation Conference. ACM, 124.

[11] Kyounghoon Kim, Jongeun Lee, and Kiyoung Choi. 2016. An energy-efficient random number generator for stochastic circuits. In Proceedings of the 201621 st Asia and South Pacific Design Automation Conference (ASP-DAC). IEEE, 256-261.

[12] Yann LeCun, Léon Bottou, Yoshua Bengio, and Patrick Haffner. 1998. Gradient-based learning applied to document recognition. Proceedings of the IEEE 86, 11 (1998), 2278-2324.

[13] Yann LeCun and Corinna Cortes. 2010. MNIST handwritten digit database. AT\&T Labs [Online]. Retrieved from http:// yann.lecun.com/exdb/mnist.

[14] Vincent T. Lee, Armin Alaghi, John P. Hayes, Visvesh Sathe, and Luis Ceze. 2017. Energy-efficient hybrid stochasticbinary neural networks for near-sensor computing. In Proceedings of the 2017 Design, Automation \& Test in Europe Conference \& Exhibition (DATE). IEEE, 13-18.

[15] Bingzhe Li, M. Hassan Najafi, and David J. Lilja. 2015. An FPGA implementation of a restricted Boltzmann machine classifier using stochastic bit streams. In 2015 IEEE 26th International Conference on Application-specific Systems, Architectures and Processors (ASAP). IEEE, 68-69.

[16] Bingzhe Li, M. Hassan Najafi, and David J. Lilja. 2016. Using stochastic computing to reduce the hardware requirements for a restricted Boltzmann machine classifier. In Proceedings of the 2016 ACM/SIGDA International Symposium on Field-Programmable Gate Arrays. ACM, 36-41.

[17] B. Li, M. H. Najafi, B. Yuan, and D. J. Lilja. 2018. Quantized neural networks with new stochastic multipliers. In Proceedings of the 2018 19th International Symposium on Quality Electronic Design (ISQED). 376-382. DOI : https://doi org/10.1109/ISQED.2018.8357316

[18] Bingzhe Li, Yaobin Qin, Bo Yuan, and David J. Lilja. 2017. Neural network classifiers using stochastic computing with a hardware-oriented approximate activation function. In Proceedings of the IEEE International Conference on Computer Design (ICCD’17). IEEE, 97-104.

[19] Ji Li, Zihao Yuan, Zhe Li, Caiwen Ding, Ao Ren, Qinru Qiu, Jeffrey Draper, and Yanzhi Wang. 2017. Hardwaredriven nonlinear activation for stochastic computing based deep convolutional neural networks. arXiv preprint arXiv:1703.04135.

[20] Peng Li, David J. Lilja, Weikang Qian, Kia Bazargan, and Marc D. Riedel. 2014. Computation on stochastic bit streams digital image processing case studies. IEEE Transactions on Very Large Scale Integration (VLSI) Systems 22, 3 (2014), 449-462.

[21] Peng Li, David J. Lilja, Weikang Qian, Marc D. Riedel, and Kia Bazargan. 2012. Logical computation on stochastic bit streams with linear finite state machines. IEEE Transactions on Computers 63, 6 (2012), 1474-1486.

[22] Peng Li, Weikang Qian, Marc D. Riedel, Kia Bazargan, and David J. Lilja. 2012. The synthesis of linear finite state machine-based stochastic computational elements. In 2012 17th Asia and South Pacific Design Automation Conference (ASP-DAC). IEEE, 757-762.

[23] Zhe Li, Ao Ren, Ji Li, Qinru Qiu, Yanzhi Wang, and Bo Yuan. 2016. DSCNN: Hardware-oriented optimization for stochastic computing based deep convolutional neural networks. In Proceedings of the IEEE 34th International Conference on Computer Design (ICCD'16). IEEE, 678-681.

[24] Zhe Li, Ao Ren, Ji Li, Qinru Qiu, Bo Yuan, Jeffrey Draper, and Yanzhi Wang. 2017. Structural design optimization for deep convolutional neural networks using stochastic computing. In Proceedings of the 2017 Design, Automation \& Test in Europe Conference \& Exhibition (DATE). IEEE, 250-253.

[25] M. Hassan Najafi, Peng Li, David J. Lilja, Weikang Qian, Kia Bazargan, and Marc Riedel. 2017. A reconfigurable architecture with sequential logic-based stochastic computing. ACM fournal on Emerging Technologies in Computing Systems ( FETC) 13, 4 (2017), 57.

[26] M. Hassan Najafi, David J. Lilja, Marc Riedel, and Kia Bazargan. 2016. Polysynchronous stochastic circuits. In Proceedings of the 2016 21st Asia and South Pacific Design Automation Conference (ASP-DAC). IEEE, 492-498.

[27] Amos R. Omondi and Jagath Chandana Rajapakse. 2006. FPGA Implementations of Neural Networks. Vol. 365. Springer.

[28] Weikang Qian and Marc D. Riedel. 2010. Synthesizing logical computation on stochastic bit streams. Submitted to Communications of the ACM (2010).

[29] Weikang Qian, Chen Wang, Peng Li, David J. Lilja, Kia Bazargan, and Marc D. Riedel. 2012. An efficient implementation of numerical integration using logical computation on stochastic bit streams. In Proceedings of the IEEE/ACM International Conference on Computer-Aided Design (ICCAD'12). IEEE, 156-162.

[30] David Silver, Aja Huang, Chris J. Maddison, Arthur Guez, Laurent Sifre, George Van Den Driessche, Julian Schrittwieser, Ioannis Antonoglou, Veda Panneershelvam, Marc Lanctot, et al. 2016. Mastering the game of Go with deep neural networks and tree search. Nature 529, 7587 (2016), 484-489. 
[31] Karen Simonyan and Andrew Zisserman. 2014. Very deep convolutional networks for large-scale image recognition. arXiv preprint arXiv:1409.1556.

[32] James E. Stine, Ivan Castellanos, Michael Wood, Jeff Henson, Fred Love, W. Rhett Davis, Paul D. Franzon, Michael Bucher, Sunil Basavarajaiah, Julie Oh, et al. 2007. FreePDK: An open-source variation-aware design kit. In Proceedings of the IEEE International Conference on Microelectronic Systems Education (MSE'07). IEEE, 173-174.

[33] Christian Szegedy, Wei Liu, Yangqing Jia, Pierre Sermanet, Scott Reed, Dragomir Anguelov, Dumitru Erhan, Vincent Vanhoucke, and Andrew Rabinovich. 2015. Going deeper with convolutions. In Proceedings of the IEEE Conference on Computer Vision and Pattern Recognition. 1-9.

Received February 2018; revised July 2018; accepted October 2018 